\title{
Molecular diagnostics in periprosthetic joint infection.
}

\author{
Javad Parvizi \\ Thomas Jefferson University Hospital \\ Lesley Walinchus \\ Thomas Jefferson University Hospital \\ Bahar Adeli \\ Thomas Jefferson University Hospital
}

Follow this and additional works at: https://jdc.jefferson.edu/orthofp

Part of the Orthopedics Commons

Let us know how access to this document benefits you

\section{Recommended Citation}

Parvizi, Javad; Walinchus, Lesley; and Adeli, Bahar, "Molecular diagnostics in periprosthetic joint infection." (2011). Department of Orthopaedic Surgery Faculty Papers. Paper 33.

https://jdc.jefferson.edu/orthofp/33

This Article is brought to you for free and open access by the Jefferson Digital Commons. The Jefferson Digital Commons is a service of Thomas Jefferson University's Center for Teaching and Learning (CTL). The Commons is a showcase for Jefferson books and journals, peer-reviewed scholarly publications, unique historical collections from the University archives, and teaching tools. The Jefferson Digital Commons allows researchers and interested readers anywhere in the world to learn about and keep up to date with Jefferson scholarship. This article has been accepted for inclusion in Department of Orthopaedic Surgery Faculty Papers by an authorized administrator of the Jefferson Digital Commons. For more information, please contact: JeffersonDigitalCommons@jefferson.edu. 


\title{
As submitted to:
}

\section{International Journal of Artificial Organs}

\section{Molecular diagnostics in periprosthetic joint infection}

\section{Volume 34, Issue 9, September 2011, Pages 847-855 \\ DOI: 10.5301/ijao.5000054}

\section{Javad Parvizi, Lesley Walinchus, Bahar Adeli}

The Rothman Institute of Orthopaedics, Thomas Jefferson University Hospital, Philadelphia, Pennsylvania - USA

\begin{abstract}
Periprosthetic joint infection (PJI) is a significant and costly challenge to the orthopedic community. The lack of a gold standard for diagnosis remains the biggest obstacle in the detection and subsequent treatment of PJI. Molecular markers in the serum and joint fluid aspirate hold immense promise to enhance the development of a firm diagnostic criterion. The primary goal is one marker with high sensitivity and specificity. Here, we review our current research efforts in the field of molecular markers: specifically, C-reactive protein, erythrocyte sedimentation rate, white blood cells, and leukocyte esterase. Each marker has been studied to determine its sensitivity, specificity, and positive and negative predictive values in diagnosing PJI.
\end{abstract}


KEY WORDS: Periprosthetic join infection, Joint arthroplasty, C-reactive protein, Leukocyte esterase, Synovial fluid aspirate

Introduction

Periprosthetic joint infection (PJI) poses a significant challenge to the orthopedic community. As the number of joint replacement surgeries in the United States continues to climb, so does the threat of infection associated with these operations. PJI has a prevalence of $1 \%$ to $4 \%$ after primary total knee arthroplasty (TKA) (1-4) and $1 \%$ to $2 \%$ after primary total hip arthroplasty (THA) $(5,6)$. At our institution, infection remains the most common cause of failure of total joint arthroplasty (TJA). As indicated by our institutional database of 20,046 hips and knee arthroplasties, $3.7 \%$ of patients required re-operation with infection as the leading cause.

The major dilemma facing the orthopedic community remains the absence of a gold standard for the diagnosis of PJI (7). Currently, a large number of tests are available and many are used in combination. Studies that aim to assess the value of each of these tests individually calculate their sensitivity, specificity, or accuracy in the detection of infection and most commonly utilize either intraoperative culture, histology results, or a combination of these parameters as the reference criterion for a comparison in diagnosis (8-13). The rate of false positives $(5-37 \%)(2,14-18)$ and false negatives $(2-18 \%)(19-23)$ of tissue and fluid culture 
decreases their accuracy in the diagnosis of PJI. A new standard that is accurate and efficient is needed in order to minimize a delay in diagnosis, prevent misdiagnosis, and provide the best treatment option in each case.

Ambiguous diagnosis in PJI cases creates significant psychological stress for both patients and surgeons looking for the best treatment options for these revision arthroplasty cases. Moreover, the financial burden of a revision arthroplasty is increased when the revision operation is for PJI, as these cases are estimated to cost $\$ 60,000$ more than mechanical revisions (24). Revisions associated with infection can lead to a higher number of hospitalizations, more days in the hospital, more operations, more outpatient visits, and also increased outpatient charges compared to revisions associated with mechanical failure (24).

Proper surgical planning for revision arthroplasty relies not only on an accurate diagnosis of infected joints, but also on isolation of the causative organism, as treatment varies accordingly. The issue of antibiotic resistance is of considerable concern due to the increase in PJI cases caused by resistant organisms. There is a higher cost associated with the treatment of infections with methicillin-resistant organisms. The cost of antibiotic resistance averages $\$ 107,264$ per case versus $\$ 68,053$ in each PJI case caused by non-resistant strains (24). Surgeons at the Rothman Institute have long been focused on the challenges of PJI, and have implemented an extensive strategy in order to combat these issues on several fronts. Discovery and utilization of molecular markers that can serve as a diagnostic tool in PJI has become the aim of research. The development of firm diagnostic criteria in order to properly identify and treat periprosthetic joint infections remains paramount. 


\section{CHALLENGES OF PERIPROSTHETIC JOINT INFECTION}

C-reactive protein (CRP) and erythrocyte sedimentation rate (ESR) analyses are currently utilized in many orthopedic practices as standard serology tests aimed at assessing joint infection. In 2009, threshold levels, with optimal sensitivity and specificity, were determined that significantly aided the clinical diagnosis of infection in hip revision cases. Based on available literature, this was the first study of its kind to statistically determine an ideal cut-off value for ESR and CRP. Patients in this study were diagnosed with PJI if they met our institutional criteria for the diagnosis of infection. Using our infection database at the Rothman Institute, serology data was retrospectively collected and examined for ESR and CRP in 479 patients $(26.5 \%$ infected). Of the infected population, $28 \%$ underwent irrigation and debridement with retention of components, $48 \%$ required two-stage resection arthroplasty for underlying infection, and $13 \%$ received a one-stage reimplantation (25). The remaining $11 \%$ were patients who were later diagnosed as infected after an original revision for mechanical failure.

In order to assess the accuracy of ESR and CRP tests in the diagnosis of PJI, receiver operating-characteristic curves (ROC) were calculated for each test. ROC allows for a visualization of the relationship between true-positives (sensitivity) and false-positives (1specificity). Area under the curve (AUC) is determined and is considered a measurement of accuracy for each test. An AUC equal to 1 denotes 100\% sensitivity and specificity, or an ideal test. An AUC $>0.9$ is considered an excellent test, while an AUC $<0.5$ is less helpful (25). In order to determine the diagnostic cut off levels of ESR and CRP, values corresponding to the points on the ROC curves nearest the upper left hand corner of the graph were chosen to 
establish an optimal balance between sensitivity and specificity in diagnosing PJI. These values were determined to be $31 \mathrm{~mm} / \mathrm{h}(\mathrm{ESR})$ and $20.5 \mathrm{mg} / \mathrm{l}$ (CRP) (25) (Fig 1).

The next phase involved evaluation and comparison of sensitivity, specificity, positive and negative predictive values for cut-offs frequently used in literature $(30 \mathrm{~mm} / \mathrm{h} \mathrm{ESR}$ and 10 mg/l CRP) with ROC-determined cut-off values (31 mm/h ESR and $20.5 \mathrm{mg} / \mathrm{l} \mathrm{CRP})$. When using the ROC-determined cut-off value of the two tests in combination (either/or scenario), we were able to improve sensitivity and negative likelihood ratio compared to both tests being used separately (Tab. I, Fig. 1). The overall success rate increases when the two tests are used in combination as the rate of false negatives from misdiagnosed cases drops to $2.7 \%$.

\begin{tabular}{l|l} 
ARBITRARILY CHOSEN FROM \\
LITERATURE TO DETERMINE
\end{tabular}

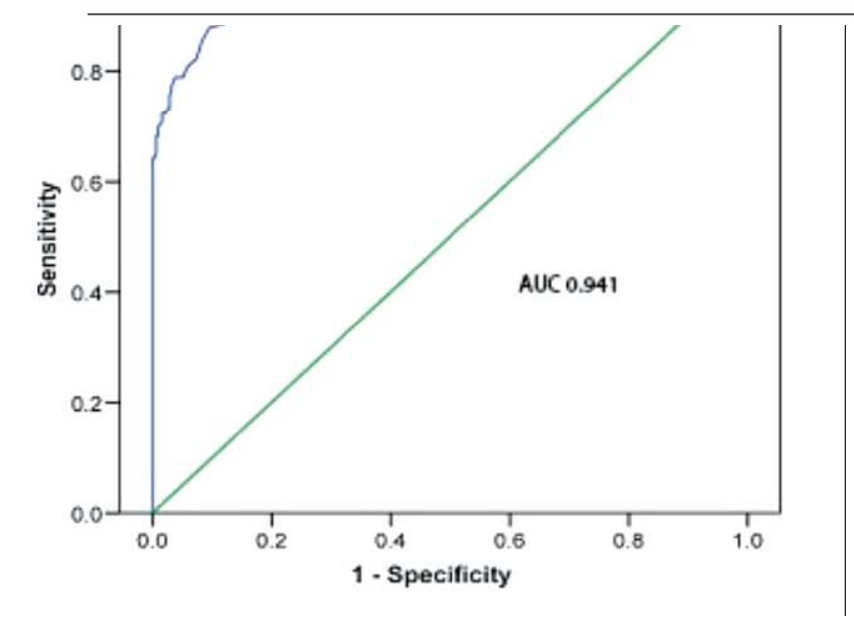

ESR AND CRP 
Fig. 1 - Receiver operating-characteristic curves for erythrocyte sedimentation rate (ESR) and C-reactive protein (CRP).

\section{ESTABLISHMENT OF A DIAGNOSTIC CRITERION}

After statistically defining ESR and CRP cut-off values, research efforts were focused on utilizing molecular diagnostics to create a diagnostic criterion for PJI. In a retrospective study, data was collected on 172 revision TKA cases at our institution between April 2002 and November 2009. These cases were used to evaluate the variation among six published sets of diagnostic criterion, which was then compared to a newly proposed set of criteria $(8 \neg 12,26)$.

Overall, there were 41 cases (24\%) where infection diagnosis conflicted between studies (27). 
According to the proposed new diagnostic criteria, PJI was suspected when one of the four parameters listed in Table II showed a positive result.

The results of our study showed an infected/uninfected ratio of 92/80. There was a large variance in sensitivity (54ᄀ100\%), specificity (39-100\%), and accuracy (53-100\%) when referenced against each of the previously established criterion (27). Interestingly, five cases were identified as infected by our proposed criteria while at least 4 of the existing sets had labeled it as uninfected. These five cases were culture negative and had no observed purulence or sinus tract intraoperatively. They were only diagnosed as infected because of elevated molecular markers from serum or synovial fluid. Three of these five had other complicating inflammatory or systemic issues (sickle cell anemia, chronic renal failure, and systemic lupus erythematosus) that contributed to the elevated levels. One of these five received a subsequent irrigation and debridement for wound dehiscence and he-matoma 2 weeks after revision. This patient's wound failed to close, and had a hematoma and necrotic tissue in superficial and deep tissue with no purulence and negative cultures. The last patient received an 8-week course of antibiotics and lifetime oral antibiotics due to coagulase-negative Staphylococcus growth in broth from preoperative joint fluid and intraoperative joint fluid during a revision to a total femur replacement. After 1 year follow-up this patient still had elevated ESR and CRP but reported no complications (27).

\section{Table II - PROPOSED NEW DIAGNOSTIC CRITERIA}

Periprosthetic Infection is present if one of the following four criteria are present:

1) Draining sinus tract

2) Positive culture on solid medium ( $>5$ colonies) 
3) Purulence seen intraoperatively

4) Three abnormal values out of following four:

a) $\mathrm{ESR} \geq 30 \mathrm{~mm} / \mathrm{hr}$ (serum)

b) C-reactive proteins $\geq 1 \mathrm{mg} / \mathrm{dL}$ (serum)

c) WBC $>1,760$ cells $/ \mu \mathrm{l}$ for acute PJI or 10,700 cells/ $\mu \mathrm{l}$ for chronic PJI (aspirate)

d) Neutrophil percentage $>73 \%$ for chronic PJI or $>89 \%$ for acute PJI (aspirate)

The discrepancy between definitions of infection serves to highlight the importance of preoperative identification of PJI using our new criteria. In order to properly plan and treat revision patients, specific and firm universal criteria must be put in place. We believe that a common set of diagnostic criteria that accounts for aspirate analysis will improve the treatment of PJI.

Shortcomings of available tests for the diagnosis of PJI motivated members of the orthopedic community to focus research efforts on the development of more accurate diagnostic criteria. Most tests are either expensive (radiology scans), subjective in their interpretation (frozen sections), not specific enough (ESR/CRP/WBC counts in serum), or simply timeconsuming (culture). In December of 2010, a collaboration between several orthopedic institutions across the country resulted in the establishment of an official working guideline for PJI. This algorithm will be a work in progress as the findings of various investigations allow for a more specific tool to increase efficiency and accuracy of diagnosis. 
Serum ESR and CRP first showed promise as molecular markers for diagnosis of PJI. However, exploration of these and other markers in the synovial fluid became the focus of research efforts aimed at finding a more specific diagnostic modality. At the Rothman Institute, synovial fluid samples were prospectively collected from 74 cases of revision hip or knee arthroplasty for septic or aseptic failure (28). Samples were shipped in bulk to Rules-Based Medicine (RBM) (Austin, TX) in order to detect and quantify the concentration of 46 inflammatory proteins in each sample. RBM assayed our samples according to their Human Inflammation Multi-analyte Profiling (MAP) protocols using a multiplex ELISA assay. To determine whether or not these samples were infected, the aforementioned institutional diagnostic criteria were used to classify each case. Thirty-one samples were classified as infected (41.9\%) and 43 as not infected (58.1\%) according to these criteria (28). Receiver operating characteristic (ROC) curves were used to investigate sensitivity, specificity, and determine the diagnostic value of each protein marker. The analysis of these 74 samples yielded 18 proteins with AUC $>0.7$ which denotes at least fair diagnostic value (Tab. III).

Identifying these anti-inflammatory proteins brings to light two important results. First, inflammatory proteins can be found in the synovial fluid in appreciable and quantifiable concentrations. Second, the variable level of concentrations of these markers parallels the presence or absence of PJI (28). The results of this study confirmed several similar conclusions regarding infection-related biomarkers in a recent study by Deirmengian et al (29) which used different immunoassays and methods to investigate 17 similar biomarkers. A fair comparison of only 6 proteins could be established and IL- 6 bore the most significant link to infected cases. The promising results obtained from the analysis of Rules Based Medicine's multiplex ELISA further 
focused research goals on investigation of those five proteins with AUC $>0.9$.

TABLE III - AUC ANALYSIS OF MOLECULAR MARKERS IN SYNOVIAL FLUID

\begin{tabular}{|c|c|c|}
\hline AUC between $0.7-0.79$ & AUC between $0.80-0.89$ & AUC between 0.9-0.99 \\
\hline - interleukin (IL)-1 receptor antagonist & - interferon- $\gamma$ & $\cdot$ IL-6 $(A \cup C=0.95)$ \\
\hline$\cdot I L-1 \beta$ & - TNF- $\beta$ & $\cdot \operatorname{IL}-8(A \cup C=0.94)$ \\
\hline$\cdot \mathrm{IL}-5$ & - von Willebrand factor & $\cdot \operatorname{CRP}(\mathrm{AUC}=0.92)$ \\
\hline tumor necrosis factor (TNF)- $\alpha$ & - matrix metalloproteinase-3 & $\alpha-2$ macroglobulin $(A \cup C=0.92)$ \\
\hline - TNF receptor-like 2 & - tissue inhibitor of metalloproteinase-1 & - Vascular Endothelial Growth Factor \\
\hline - Macrophage inflammatory protein $-1 \beta$ & & $(\mathrm{VEG}-\mathrm{F})(\mathrm{AUC}=0.90)$ \\
\hline \multicolumn{3}{|l|}{$\beta-2$ microglobulin } \\
\hline - Vascular cell adhesion molecule-1 & & \\
\hline
\end{tabular}

\section{MOLECULAR MARKERS IN SYNOVIAL FLUID}

Several studies have taken a closer look at synovial fluid for biomarkers in PJI. Each study proposed slightly varying cut off values for normal ranges of ESR, CRP, WBC, or PMN\% (3133). This was the impetus for our multi-institutional collaborative effort with Rush University Medical Center (Chicago, IL, USA) and The Center for Adult Joint Reconstruction (Newton, MA, USA) aimed to investigate specific inflammatory markers for PJI in synovial fluid and resulted in a Mark Coventry Award publication. A retrospective review was conducted of 11,964 primary TKAs performed on 9,826 patients at these institutions between April 1999 and December 2008. Of the 146 cases identified as having received a knee aspiration within 6 weeks of surgery, there were 19 cases classified as infected due to positive cultures or gross purulence (30). Each sample was evaluated for white blood cell (WBC) count and differential as well as CRP and/or ESR levels in order to classify infected from non-infected cases.

The results of this collaborative cohort indicate a significantly elevated difference between the infected group compared to the noninfected group for synovial WBC count ( $\mathrm{p}$ $<0.0001)$, PMN\% ( $\mathrm{p}=0.0314)$, and CRP values $(\mathrm{p}=0.0042)$. No significant difference in ESR 
levels was found between the two cohorts $(\mathrm{p}=0.4626)$ (30) (Tab. IV). Ultimately, this study showed the diagnostic value of synovial WBC as a reliable marker for PJI during the early postoperative period.

\section{C-REACTIVE PROTEIN (CRP)}

Ongoing efforts aimed at finding a gold standard diagnostic test have brought great attention to CRP in synovial fluid. This promising biomarker has potential to serve as a low-cost diagnostic tool for PJI. CRP is a common serology test that is easily analyzed under routine hospital laboratory conditions.

RBM analysis of CRP showed a threshold level of $3.605 \mathrm{mg} / \mathrm{l}$ in synovial fluid analysis with a sensitivity of $87.1 \%$, specificity of $97.7 \%$, PPV of $96.4 \%$, NPV of $91.5 \%$ and an accuracy of $93.3 \%$ (28). Based on this data, our goal was to investigate whether the accuracy of standard CRP assay for detection of PJI could be improved by measuring CRP in synovial fluid. Synovial fluid specimens were collected in the operating room before arthroplasty from 72 cases of revision total knee arthroplasty in order to calculate the concentration of synovial CRP to determine the sensitivity, specificity, PPV, and NPV (34). These samples were all labeled and classified as infected or uninfected based on our institutional criteria for diagnosis of PJI. All 72 (29 infected, 43 uninfected) samples that were sent to RBM to be run on a multiplex immunoassay platform and 15 samples were also analyzed using CRP ELISA kit (10 infected and 5 uninfected) (GenWay Biotech, San Diego, CA, USA). All preoperative serum CRP samples (68 samples; 29 infected, 39 uninfected) were analyzed by review of data from hospital records. For each assay, sensitivity, specificity, and ROC curve analyses was generated using our institutional diagnostic criteria (34) (Tab. V). 
The findings of this study indicate that CRP concentrations in the synovial fluid rather than in blood serum improves the diagnostic accuracy of this test in identifying PJI, making CRP a promising biomarker in this field. The results spurred further study of CRP using larger sample sizes as well as the expansion of the current cohort to include patients with failed hip arthroplasties. In our prospective study, synovial fluid samples collected from patients undergoing primary and revision TJA are investigated for any correlation between synovial and serum CRP levels as well as white blood cell count and differential. Interim analysis of 97 synovial fluid samples from 97 patients, 33 diagnosed with PJI and 64 not infected, shows statistically significant differences between the groups for serum CRP, synovial fluid CRP, ESR, synovial WBC count, and neutrophil differential $(\mathrm{p}<0.001)$. The mean aseptic synovial fluid CRP value was $1.64 \mathrm{mg} / \mathrm{l}$ while the mean septic synovial fluid CRP value was $46.27 \mathrm{mg} / \mathrm{l}$ ( $\mathrm{p}<0.0001$ ). An ROC curve shows $84.8 \%$ sensitivity, 95.5\% specificity, at a CRP synovial fluid threshold of $9.5 \mathrm{mg} / \mathrm{l}$ and an AUC of 0.93. Although this data is not finalized, we are hopeful that CRP will continue to show promise for the development of a quick, easy, and low-cost molecular diagnostic tool.

TABLE IV- SYNOVIAL FLUID MOLECULAR MARKERS FROM COLLABORATIVE DATA

\begin{tabular}{lcc}
\hline Diagnostic Measure & Infected & Noninfected \\
\hline ESR (mm/hour) & $80 \pm 29(38-140)$ & $75 \pm 30(1-140)$ \\
CRP (mg/dL) & $171 \pm 127(29-490)$ & $88 \pm 75(4-382)$ \\
Synovial fluid \%PMN & $89.6 \pm 20.6(6-99)$ & $76.9 \pm 21.2(5-100)$ \\
Synovial fluid WBC count (cells/ $\mathrm{LL})$ & $92,600 \pm 127,000(3520-570,000)$ & $4200 \pm 5700(0-41,000)$ \\
\hline Values are expressed as mean \pm SD, with range in parentheses; ESR = erythrocyte sedimentation rate; CRP = C-reactive protein; \%PMN = percentage of polymorpho- \\
nuclear cells; WBC = white blood cell.
\end{tabular}

\section{LEUKOCYTE ESTERASE: A POTENTIAL DIAGNOSTIC TOOL}


One of the most exciting molecular markers we are working on is leukocyte esterase (LE). We hypothesized that neturophils recruited into an infected joint would release LE and its presence would suggest PJI. Between May of 2007 and April of 2009, the following two study cohorts were evaluated: (i) 108 patients (30 infected, 78 uninfected) undergoing revision TKA at the Rothman Institute, for whom aspirations were done intraoperatively, just prior to arthrotomy; and (ii) 17 (5 infected, 12 uninfected) patients undergoing a PJI workup with knee aspirations collected at the time of the clinic visit (35). All patients were classified as infected/uninfected based on the previously discussed, newly proposed institutional criteria.

In order to measure the LE presence in joint fluid, a standard chemical test strip that contained seven reagent pads: $\mathrm{pH}$, glucose, ketones, protein, nitriles, and blood was used (Roche Chemstrip ${ }^{\circ} 7$ Urine Test Strip, Roche Diagnostics, Indianapolis, IN, USA). The colorimetric change in LE reagent pad occurs via chemical reaction, and results in a purple color which is read as negative (white), trace (slightly purple), + (light purple), or ++ (dark purple). The samples were applied directly to the strips and results were read after a minute. The use of these test trips enables results to be available rapidly and within the clinic setting. Table VI dis-plays the results obtained when ++ or + was deemed positive.

Expansion of this data set is part of current research efforts at our institution. Presently, the LE strip test boasts high sensitivity and specificity for diagnosis of PJI. Our results approach and even exceed those of several standard tests that are currently implemented $(7,9)$. Additionally, this data is was investigated for any correlation with typical serology findings associated with PJI such as ESR, CRP, fluid WBC, and polymorphonuclear leukocyte counts (PMN). Strongest correlation was found with PMN \% (r=0.7769), as expected (35). 
The ease, speed, and relative low-cost of the LE strip test make it very desirable as an adjunct molecular diagnostic tool for orthopedic surgeons. While the final role of this leukocyte esterase test is to be determined, the high PPV of a ++ result (strongly positive) and the high NPV of a negative/trace result (negative) indicate its value as a diagnostic tool which can serve to increase preoperative planning because of a clear diagnosis. Future directions for this study aim to expand the cohort, decrease reader bias using electronic readers, and investigate ways to include samples that are contaminated with blood which can currently skew results of this colorimetric test. Improvement in these areas would hopefully enable the use of leukocyte esterase to be utilized in the diagnosis of PJI.

TABLE V - SUMMARY OF THREE CRP ASSAY RESULTS

\begin{tabular}{|c|c|c|c|c|c|c|c|}
\hline Fluid & Assay & $\begin{array}{c}\text { AUC } \\
(95 \% \mathrm{Cl})\end{array}$ & $\begin{array}{l}\text { Threshold } \\
\text { (mg/L) }\end{array}$ & $\begin{array}{l}\text { Sensitivity } \\
\text { (\%) }\end{array}$ & $\begin{array}{l}\text { Specificity } \\
\text { (\%) }\end{array}$ & $\begin{array}{l}\text { PPV } \\
(\%)\end{array}$ & $\begin{array}{l}\text { NPV } \\
(\%)\end{array}$ \\
\hline \multirow[t]{2}{*}{ Synovial fluid } & $\begin{array}{l}\text { Individual ELISA } \\
\qquad(\mathrm{N}=15)\end{array}$ & $\begin{array}{c}0.84 \\
(0.63-1.04)\end{array}$ & 0.06 & 70.0 & 100.0 & 100.0 & 62.5 \\
\hline & $\begin{array}{l}\text { Multiplex ELISA } \\
\qquad(\mathrm{N}=72)\end{array}$ & $\begin{array}{c}0.92 \\
(0.85-0.99)\end{array}$ & 3.6 & 86.2 & 97.7 & 96.2 & 91.3 \\
\hline \multirow[t]{2}{*}{ Serum } & $\begin{array}{l}\text { Clinical laboratory assay } \\
\qquad(N=68)\end{array}$ & $\begin{array}{c}0.89 \\
(0.80-0.97)\end{array}$ & 16.5 & 72.4 & 94.9 & 91.3 & 82.2 \\
\hline & & & 9.5 & 82.8 & 84.6 & 80.0 & 86.8 \\
\hline
\end{tabular}

\section{MISSING THE MARK: CULTURE-NEGATIVE INFECTIONS}

It is important to mention a specific subset of patients diagnosed with PJI at our institution who lack a positive culture result. Without a causative organism, a significant road block is created not only in the surgical planning but also in the choice of anti-infective agent used by the surgeon. Even though there is a significant success rate associated with current treatments like irrigation and debridement (I\&D), two-stage or one-stage revision, these cases must be 
considered in order to determine the best treatment plan. A review of 651 cases of PJI treated at our institution between 1999 and 2007 revealed 48 cases of PJI that were culture- negative. These cases were compared to culture-positive cases treated during the same time frame. Surgical intervention for these 48 cases included: I\&D (13), one-stage exchange arthroplasty (3), and planned two-stage exchange arthroplasty (6) (Tab. VII). There is reason to believe that the most likely cause for these suboptimal results for culture-negative PJI cases stems from an inability to isolate a pathogen from conventional culture. This ongoing study serves to underscore the importance of additional efforts to identify fungi or atypical pathogens that could be the cause of CN PJI.

One such effort focused on the use of a new molecular diagnostic tool called IBIS T5000 Biosensor in an attempt to uncover a causative organism in $\mathrm{CN}$ cases of PJI. This technology utilizes pan-genomic primers in a series of polymerase chain reactions to identify known pathogens and virulence genes. Using mass spectrometry to asses the base composition of an identified region of DNA, this technology identifies specific genes, such as the mecA gene for methicillin resistance, giving surgeons important information about the type of pathogen isolated from the joint. These results, combined with the PCR results, are then compared to a database to match them with the closest possible microorganism (12). We prospectively collected intraoperative synovial fluid samples from 80 patients (65 revision total knee and 15 revision hip arthroplasty) and analyzed them with IBIS as well as conventional culture. The surgeon determined 23 of the cases to be infected and 57 as uninfected or aseptic revisions. Eighteen of the 23 infected cases were culture-positive, and IBIS isolated the same organism in 17 of these cases. Though the remaining 5 infected cases were culture negative, IBIS isolated an organism in 4 of them. Furthermore, IBIS found a pathogen in 50 of the 57 aseptic revision cases. If one uses 
a positive culture to indicate infection, then $87.7 \%$ of the so-called aseptic revisions may indeed be missed infections. Due to its increased sensitivity compared to culture, we believe that IBIS may be of great value in isolating a microorganism in specifc cases with either negative cultures or abnormal serology and/or cell count. Furthermore, by providing a plethora of information, such as the discovery of new genes and mutations within species, IBIS can help to better understand the diagnosis of treatment of patients with PJI.

TABLE VI - SUMMARY OF LEUKOCYTE ESTERASE RESULTS

\begin{tabular}{|c|c|c|c|c|c|}
\hline Cohort & Positive Results: & $\begin{array}{c}\text { Sensitivity (\%) } \\
(95 \% \mathrm{Cl})\end{array}$ & $\begin{array}{c}\text { Specificity (\%) } \\
(95 \% \mathrm{Cl})\end{array}$ & $\begin{array}{l}\text { PPV (\%) } \\
(95 \% \mathrm{Cl})\end{array}$ & $\begin{array}{l}\text { NPV (\%) } \\
(95 \% \mathrm{Cl})\end{array}$ \\
\hline \multirow[t]{2}{*}{ Revision knees ( $\mathrm{N}=108$ ) } & ++ & $\begin{array}{c}80.6 \\
(61.9-91.9)\end{array}$ & $\begin{array}{c}100 \\
(94.5-100)\end{array}$ & $\begin{array}{c}100 \\
(83.4-100)\end{array}$ & $\begin{array}{c}93.3 \\
(85.4-97.2)\end{array}$ \\
\hline & + or ++ & $\begin{array}{c}93.5 \\
(77.2-98.8)\end{array}$ & $\begin{array}{c}86.7 \\
(77.1-92.9)\end{array}$ & $\begin{array}{c}72.5 \\
(55.9-84.9)\end{array}$ & $\begin{array}{c}97.3 \\
(89.7-99.5)\end{array}$ \\
\hline \multirow[t]{2}{*}{ Clinic samples $(\mathrm{N}=17)$} & ++ & $\begin{array}{c}80.0 \\
(29.9-98.9)\end{array}$ & $\begin{array}{c}100 \\
(69.9-100)\end{array}$ & $\begin{array}{c}100 \\
(39.6-100)\end{array}$ & $\begin{array}{c}92.3 \\
(62.1-99.6)\end{array}$ \\
\hline & + or ++ & $\begin{array}{c}100 \\
(46.3-100)\end{array}$ & $\begin{array}{c}41.7 \\
(16.5-71.4)\end{array}$ & $\begin{array}{c}41.7 \\
(16.5-71.4)\end{array}$ & $\begin{array}{c}100 \\
(46.3-100)\end{array}$ \\
\hline
\end{tabular}

TABLE VII - CULTURE-POSITIVE AND CULTURE-NEGATIVE SUCCESS RATES

\begin{tabular}{lcc}
\hline Treatment & CN success rate & CP success rate \\
\hline Irrigation \& debridement & $50 \%(n=12)$ & $45.6 \%(n=103)$ \\
Two-stage revision & $70.4 \%(n=27)$ & $80.6 \%(n=160)$ \\
\hline
\end{tabular}

\section{CONCLUSIONS}

Periprosthetic joint infection compromises the results of total joint arthroplasty, which historically has been a highly successful operation. The work investigating the molecular markers discussed in this paper aims to narrow the focus of future research in the diagnosis and treatment of PJI. Future research on PJI aims to combat current diagnostic

challenges through the development of an accurate and efficient tool. The primary goal is one marker with high sensitivity and specificity. Ongoing studies aim to expand the cohort in cases 
with C-reactive protein and leukocyte esterase analysis. Results thus far give promise that molecular markers can facilitate diagnosis of PJI as they may serve as a future gold standard modality that is rapid, inexpensive, and highly accurate.

\section{REFERENCES}

1. Della Valle CJ, Zuckerman JD, Di Cesare PE. Periprosthetic Sepsis. Clin Orthop Relat Res. 2004;420(420):26-31. doi:10.1097/00003086-200403000-00005.

2. Duff GP, Lachiewicz PF, Kelley SS. Aspiration of the knee joint before revision arthroplasty. Clin Orthop Relat Res. 1996;331(331):132-139. doi:10.1097/0000308619961000000018.

3. Peersman G, Laskin R, Davis J, Peterson M. Infection in total knee replacement: a retrospective review of 6489 total knee replacements. Clin Orthop Relat Res. 2001;392(392):1523. doi:10.1097/00003086-200111000-00003.

4. Phillips JE, Crane TP, Noy M, Elliott TS, Grimer RJ. The incidence of deep prosthetic infections in a specialist orthopaedic hospital: A 15-year prospective survey. J Bone Joint Surg Br. 2006;88-B(7):943-948. doi:10.1302/0301620X.88B7.17150.

5. Parvizi J, Ghanem E, Azzam K, Davis E, Jaberi F, Hozack W. Periprosthetic infection: are current treatment strategies adequate? Acta Orthop Belg. 2008;74(6):793-800.

6. Sculo TP. The economic impact of infected total joint arthroplasty. Instr Course Lect. $1993 ; 42: 349-351$. 
7. Bauer TW, Parvizi J, Kobayashi N, Krebs V. Diagnosis of periprosthetic infection. J Bone Joint Surg Am. 2006;88(4):869ᄀ882. doi:10.2106/JBJS.E.01149.

8. Berbari EF, Hanssen AD, Duffy MC, et al. Risk factors for prosthetic joint infection: case-control study. Clin Infect Dis. 1998;27(5):1247-1254. doi:10.1086/514991.

9. Parvizi J, Ghanem E, Menashe S, Barrack RL, Bauer TW. Periprosthetic infection: what are the diagnostic challenges? J Bone Joint Surg Am. 2006;88(Suppl 4):138-147. doi:10.2106/JBJS.F.00609.

10. Parvizi J, Ghanem E, Sharkey P, Aggarwal A, Burnett RSJ, Barrack RL. Diagnosis of infected total knee: findings of a multicenter database. Clin Orthop Relat Res. 2008;466(11):2628-2633. doi:10.1007/s11999-008-0471-5.

11. Schinsky MF, Della Valle CJ, Sporer SM, Paprosky WG. Perioperative testing for joint infection in patients undergoing revision total hip arthroplasty. J Bone Joint Surg Am. 2008;90(9):1869-1875. doi:10.2106/JBJS.G.01255.

12. Spangehl MJ, Masri BA, O'Connell JX, Duncan CP. Prospec-tive analysis of preoperative and intraoperative investigations for the diagnosis of infection at the sites of two hundred and two revision total hip arthroplasties. J Bone Joint Surg Am. 1999;81(5):672-683.

13. Trampuz A, Piper KE, Jacobson MJ, et al. Sonication of removed hip and knee prostheses for diagnosis of infection. N Engl J Med. 2007;357(7):654-663. doi:10.1056/NEJMoa061588. 
14. Abdul-Karim FW, McGinnis MG, Kraay M, Emancipator SN, Goldberg V. Frozen section biopsy assessment for the presence of polymorphonuclear leukocytes in patients undergoing revision of arthroplasties. Mod Pathol. 1998;11(5):427-431.

15. Lachiewicz PF, Rogers GD, Thomason HC. Aspiration of the hip joint before revision total hip arthroplasty. Clinical and laboratory factors influencing attainment of a positive culture. J Bone Joint Surg Am. 1996;78(5):749-754.

16. Lonner JH, Desai P, Dicesare PE, Steiner G, Zuckerman JD. The reliability of analysis of intraoperative frozen sections for identifying active infection during revision hip or knee arthroplasty. J Bone Joint Surg Am. 1996;78(10):1553-1558.

17. Mirra JM, Marder RA, Amstutz HC. The pathology of failed total joint arthroplasty. Clin Orthop Relat Res. 1982;(170):175ᄀ

18. Padgett DE, Silverman A, Sachjowicz F, Simpson RB, Rosen-berg AG, Galante JO. Efficacy of intraoperative cultures obtained during revision total hip arthroplasty. J Arthroplasty. 1995;10(4):420-426. doi:10.1016/S0883-5403(05)80140-8.

19. Athanasou NA, Pandey R, de Steiger R, Crook D, Smith PM. Diagnosis of infection by frozen section during revision arthroplasty. J Bone Joint Surg Br. 1995;77(1):28-33.

20. Fehring TK, McAlister JA Jr. Frozen histologic section as a guide to sepsis in revision joint arthroplasty. Clin Orthop Relat Res. 1994;(304):229-237.

21. Feldman DS, Lonner JH, Desai P, Zuckerman JD. The role of intraoperative frozen sections in revision total joint arthroplasty. J Bone Joint Surg Am. 1995;77(12):1807-1813. 
22. Pandey R, Berendt AR, Athanasou NA; The OSIRIS Col-laborative Study Group. Oxford Skeletal Infection Research and Intervention Service. Histological and microbiological findings in non-infected and infected revision arthroplasty tissues. Arch Orthop Trauma Surg. 2000;120(10):570-574. doi:10.1007/s004020000174.

23. Tunney MM, Patrick S, Gorman SP, et al. Improved detec-tion of infection in hip replacements: A currently underestimated problem. J Bone Joint Surg Br. 1998;80(4):568-572. doi:10.1302/0301-620X.80B4.8473.

24. Bozic KJ, Ries MD. The impact of infection after total hip arthroplasty on hospital and surgeon resource utilization. J Bone Joint Surg Am. 2005;87(8):1746-1751. doi:10.2106/JBJS.D.02937.

25. Ghanem E, Antoci V Jr, Pulido L, Joshi A, Hozack W, Parvizi J.

The use of receiver operating characteristics analysis in deter-mining erythrocyte sedimentation rate and C-reactive protein levels in diagnosing periprosthetic infection prior to revision total hip arthroplasty. Int J Infect Dis. 2009;13(6):e444-e449. doi:10.1016/j.ijid.2009.02.017. 
26. Trampuz A, Hanssen AD, Osmon DR, Mandrekar J, Steckel-berg JM, Patel R. Synovial fluid leukocyte count and differential for the diagnosis of prosthetic knee infection. Am J Med. 2004;117(8):556-562. doi:10.1016/j.amjmed.2004.06.022.

27. Parvizi J, Jacovides C, Zmistowski B, Jung KA. Definition of periprosthetic joint infection: is there a consensus? Clin Orthop Relat Res. 2011;469(11):3022-3030.

28. Jacovides CL, Parvizi J, Adeli B, Jung KA. Molecular Markers for Diagnosis of Periprosthetic Joint Infection. J Arthro-plasty 2011;26(6 Suppl):99-103.

29. Deirmengian C, Hallab N, Tarabishy A, et al. Synovial fluid biomarkers for periprosthetic infection. Clin Orthop Relat Res. 2010;468(8):2017-2023. doi:10.1007/s11999010-1298-4.

30. Bedair H, Ting N, Jacovides C, et al. The Mark Coventry Award: diagnosis of early postoperative TKA infection using synovial fluid analysis. Clin Orthop Relat Res. 2011;469(1):34-40. doi:10.1007/s11999-010-1433-2. 
31. Della Valle CJ, Sporer SM, Jacobs JJ, Berger RA, Rosenberg AG, Paprosky WG.

Preoperative testing for sepsis before revision total knee arthroplasty. J Arthroplasty. 2007;22(6) (Suppl 2):90-93. doi:10.1016/j.arth.2007.04.013.

32. Mason JB, Fehring TK, Odum SM, Griffin WL, Nussman DS. The value of white blood cell counts before revision total knee arthroplasty. J Arthroplasty. 2003;18(8):1038-1043. doi:10.1016/S0883-5403(03)00448-0.

33. Ghanem E, Parvizi J, Burnett RSJ, et al. Cell count and dif-ferential of aspirated fluid in the diagnosis of infection at the site of total knee arthroplasty. J Bone Joint Surg Am. 2008;90(8):1637-1643. doi:10.2106/JBJS.G.00470.

34. Parvizi J, Jacovides C, Adeli B, Jung KA, Hozack W. CRP in joints: a molecular marker for periprosthetic joint infection? J Arthroplasty. In press.

35. Parvizi J, Jacovides C, Antoci V, Ghanem E. Diagnosis of periprosthetic joint infection: the role of a simple, yet unrecognized, enzyme. J Arthroplasty. In Press. 